\title{
Selbstkonsistenz vs. Gruppenkonformität bei Selbstaufmerksamkeit: Die Rolle des Einflusses von Ingroup vs. Outgroup
}

\author{
AmÉLIE MUMMENDEY \\ Psychologisches Institut der Universität Münster
}

Hans DieTer Mummendey

Universität Bielefeld

In einem Experiment mit 96 weiblichen Versuchsteilnehmern wurde (1) die Annahme von WICKLUND (1980) überprüft, daß Personen dann selbstkonsistent und unabhängig von der Gruppenmeinung urteilen, wenn sie zuvor ihre Meinung frei äußern konnten und sich im Zustand objektiver Selbstaufmerksamkeit befinden, (2) die Annahme geprüft, daß die Art der Beziehung zwischen Individuum (Frau) und Gruppe (Ingroup = Frauen, vs. Outgroup = Männer) für das Ausmaß der Selbstkonsistenz vs. Gruppenkonformität im Hinblick auf die soziale Einstellung «Gleichberechtigung der Frau» ausschlaggebend ist. Die Ergebnisse stützen die zweite Annahme: Es zeigte sich ein differentieller Einfluß von Ingroup vs. Outgroup auf die Einstellungsänderung nur bei fehlender Selbstaufmerksamkeit, nicht bei objektiver Selbstaufmerksamkeit. Die Wechselwirkung wurde im Rahmen der Social IdentityTheorie (TAJFEL \& TURNER) interpretiert.
In an experiment with 96 female subjects two assumptions should be tested, (1) the assumption of WICKLUND (1980) that persons' judgments are self-consistent and independent of group's attitude if they had the opportunity of free utterance of their attitudes before, and if they were in a state of objective self awareness, (2) that the kind of relation between individual (woman) and group (ingroup, i.e. women, vs. outgroup, i. e. men) is crucial for the amount of selfconsistency vs. group conformity with respect to the social attitude (equality of rights for women and men). Results are supporting the second assumption. Only under attention outside self, not under objective self awareness conditions, a differential influence of ingroup vs. outgroup upon attitude change (group conformity vs. self-consistency) was shown. This interaction effect is interpreted within the framework of social identity theory (TAJFEL \& TURNER).

\section{Problemstellung}

Die vorliegende Arbeit versucht mit experimentellen Mitteln eine Frage zu beantworten, die im Rahmen der Theorie der objektiven Selbstaufmerksamkeit von DUVAL \& WICKLUND (1972) entstanden ist: Ist bei gegebener objektiver Selbstaufmerksamkeit Selbstkonsistenz unabhängig von den Einstellungen der Gruppe wahrscheinlicher als Gruppenkonformität, oder hängt dies von der Art der Beziehung zwischen Individuum und Gruppe ab?

Die Theorie der objektiven Selbstaufmerksamkeit (objective self awareness = OSA) geht davon aus, daß ein Individuum in einen $\mathrm{Zu}$ stand geraten kann, in dem es seine Aufmerksamkeit auf die eigene Person richtet (OSA), im Unterschied zu dem gewöhnlich häufigeren Zustand, in welchem es seine Aufmerksamkeit nach außen richtet (attention outside self $=$
AOS). Wird das Individuum dazu veranlaßt, sich selbst zu beobachten (OSA), so entsteht insofern ein für die Person unangenehmer $\mathrm{Zu}$ stand, als möglicherweise Inkonsistenzen zwischen den eigenen Einstellungen und dem eigenen Verhalten wahrgenommen werden. Im Zustande der OSA würden demzufolge Tendenzen entstehen, diese wahrgenommenen Diskrepanzen zu reduzieren, d.h., Einstellungs-Verhaltens-Konsistenz herzustellen. Experimente, die im Rahmen der OSA-Theorie ausgeführt wurden, haben verschiedentlich die Tendenz zu erhöhter Selbstkonsistenz aufgezeigt (z.B. CARver, 1975; GibBons, 1978). Auf der anderen Seite postuliert die OSA-Theorie (vgl. Wicklund \& Duval, 1971; Duval, 1972, 1976), daß unter OSA-Bedingungen die soziale Konformität, d.h., die Anpassung an die Standards einer Gruppe erhöht wird, da im Zustande objektiver Selbstaufmerksamkeit Gruppen- 
Normen bewußter bzw. salienter werden als unter AOS-Bedingungen.

In seinem Beitrag für den Sammelband über Gruppeneinfluß von Paulus postuliert WICKLUND (1980), daß selbstaufmerksame Personen in Situationen, in denen Gruppenstandards salient sind, in der Regel gruppenkonform reagieren, daß sie jedoch dann eher selbstkonsistent reagieren, wenn sie ihre persönlichen Einstellungen zuvor frei geäußert haben (im Sinne eines commitment). WICKLUND zitiert hierzu die Ergebnisse einer experimentellen Untersuchung von MCCORMICK (1979), denen zufolge Individuen unter OSA eher konsistent mit ihren eigenen Einstellungen, vollständig unabhängig von der Gruppen-Einstellung («totally independent of the group's attituden; WICKLUND, 1980, p. 204) reagierten, wenn sie die eigene Einstellung zuvor frei äußern konnten.

MCCoRMick (1979) stellte in seinem Experiment Gruppenkonformität und Selbstkonsistenz entweder unter FreeChoice- oder unter Controlled-Choice-Bedingungen gegenüber, indem Versuchspersonen eine Aufgabe entweder frei wählen (〈Intellectual Games People Play) in selbstgewählter Reihenfolge spielen) oder nicht frei wählen konnten (die gleichen Spiele in einer vorgegebenen Folge, die durch die Wahlen einer Vp aus der Free-Choice-Bedingung vorgegeben war, spielen). Jeweils die Hälfte der Vpn unter beiden Bedingungen arbeitete unter OSA- bzw. AOSBedingungen. Schließ3lich wurden die Personen jeder dieser vier Bedingungskombinationen zur Hälfte einem Gruppenkonformitätseinfluß, und zur Hälfte keinem solchen Einflu $\beta$ ausgesetzt, indem mehrere vorgegebene Rangreihen der Intellectual Games zu beurteilen waren; dabei wurden unter der 〈Konformitäts〉-Bedingung die Rangreihen mit einem Bericht versehen, aus dem hervorging, daß mehrere Gruppenmitglieder (Versuchspersonen der gleichen Studentengruppe) die Rangreihen gelegt und begründet hatten, wobei das von der jeweiligen Vp am stärksten abgelehnte Spiel von der Gruppe angeblich am meisten präferiert wurde. Unter der (Konsistenz)-Bedingung waren die gleichen Spiele ohne jeden Zusatzkommentar zu beurteilen, d.h., es wurde kein Konflikt zwischen der eigenen und der angeblichen Gruppenmeinung erzeugt. Die Varianzanalysen MCCORMICKs ergaben für die abhängige Variable «Effort» (für jede Aufgabe aufgewendete Zeit) eine signifikante Wechselwirkung der Faktoren «Awareness» (OSA/AOS), «Choice» (Free Choice/No Choice) und "Group Pressure» (Konformität/Konsistenz); Personen, die ein freies Commitment eingegangen waren, erwiesen sich als konsistenter als solche, denen man keine Wahl gelassen hatte, und zwar besonders unter OSA-Bedingungen, während Personen ohne Selbstaufmerksamkeit (also unter AOS-Bedingungen) sich hinsichtlich Gruppenkonformität/Selbstkonsistenz nicht unterschieden.

In der vorliegenden Arbeit zielen wir a) auf eine teilweise Replikation des Experimentes von MCCoRMICK, teilweise stellen wir b) die Behauptung in Frage, daß unabhängig von den Einstellungen der Gruppe, die einen Einfluß ausübt, Selbstkonsistenz wahrscheinlicher ist als Gruppen konformität, wenn freie Wahlmöglichkeit und objektive Selbstaufmerksamkeit gegeben sind.

Aus sozialpsychologischer Perspektive erscheint die Aussage, daß unter den genannten Bedingungen Selbstkonsistenz vollständig unabhängig von der Gruppeneinstellung wahrscheinlicher ist, als problematisch. Der Gruppeneinfluß dürfte sich nämlich unterschiedlich auswirken je nachdem, um welche Art von beeinflussender Gruppe es sich handelt, d.h. welcher Art die Beziehungen zwischen Individuum und Gruppe tatsächlich sind bzw. welche Meinungen die Mitglieder der Gruppe vermutlich haben (vgl. beispielsweise TAJFEL \& TuRNER, 1979). Es erscheint hier z.B. von Bedeutung, ob es sich um eine einstellungsmäßigähnliche oder unähnliche Gruppe handelt, ob die Gruppe als "Ingroup» oder "Outgroup» wahrgenommen wird, usw. Wir erwarten demnach, daß der Einflu $\beta$ der Bedingungen der objektiven Selbstaufmerksamkeit durch die Art der Beziehung zwischen Individuum und Gruppe modifiziert wird und nehmen an, daß das Verhältnis von Selbstkonsistenz und Gruppenkonformität davon beeinflußt wird, ob die Person mit Standards ihrer Ingroup oder Outgroup konfrontiert wird. Zwar haben WICKLUND \& Duval (1971) bereits den Einfluß unterschiedlich attraktiver Gruppen auf die Einstellungsänderung bei mehroder weniger großer objektiver Selbstaufmerksamkeit untersucht, doch waren die dort herangezogenen, unterschiedlichen Gruppen (Studenten vs. Strafgefangene) nicht als spezifische In- bzw. Outgroups in bezug auf das Einstellungsobjekt anzusehen.

In dem folgenden Experiment sollen daher «Selbstaufmerksamkeit» (OSA/AOS), "Wahlfreiheits (Commitment/Kein Commitment) und «Gruppenstandard» (Ingroup/Outgroup/ Keine Gruppe) faktoriell variiert werden. Als abhängige Variable sollen die Konsistenzen von Präferenzurteilen zur Lösung eines sozialen Problems dienen, von dem angenommen werden kann, daß es für die Versuchspersonen von persönlicher Wichtigkeit ist. Es soll die $H y$ - 
pothese einer Wechselwirkung zwischen den Faktoren «Selbstaufmerksamkeit» und «Gruppenstandard» geprüft werden; wir nehmen an, daß es zu der von WICK LUND postulierten «Unabhängigkeit von der Gruppen-Einstellung» nur hinsichtlich Individuum-Outgroup-, nicht jedoch hinsichtlich IndividuumIngroup-Beziehungen kommt. Von vornherein erscheint diese Annahme nur für den Fall sinnvoll, daß die Personen anfangs frei wählen bzw. ein Commitment machen können, da andernfalls schwerlich von «Selbstkonsistenz» gesprochen werden kann.

\section{Methode}

Versuchsplan. In einem $2 \times 2 \times 3$-Plan (bezüglich der ersten der beiden abhängigen Variablen) bzw. einem $2 \times 2 \times 2$-Plan (bezüglich der zweiten der beiden abhängigen Variablen) mit den Faktoren «Selbstaufmerksamkeit», «Wahlfreiheit» und «Gruppenstandard» werden die Konsistenzen bzw. intraindividuellen Korrelationen von Präferenzurteilen bezüglich Vorschlägen zur Lösung des sozialen Problems «Gleichstellung der Frau» untersucht.

Versuchspersonen. Als Vpn wurden 96 Studentinnen der Universität Bielefeld angeworben und nach Zufall auf die 12 Zellen des Versuchsplans (bzw. bezüglich der zweiten abhängigen Variable auf acht Bedingungskombinationen) verteilt (vgl. Tab. 1).

Alle Vpn wurden mit dem deutlichen Hinweis angeworben, daß ausschließlich weibliche Personen benötigt würden. Die meisten Vpn studierten für das Lehramt (54), es folgten Rechtswissenschaften (12), Biologie (7), Mathematik (6), und der Rest verteilte sich auf un-

Tab. 1: Versuchsplan und Aufteilung der Vpn auf die Bedingungskombinationen.

\begin{tabular}{lllll}
\hline $\begin{array}{l}\text { Weibliche Vpn } \\
\text { (n=96) }\end{array}$ & \multicolumn{3}{l}{ "Commitment» } & $\begin{array}{l}\text { «Kein Com- } \\
\text { mitment» }\end{array}$ \\
\cline { 2 - 6 } & OSA & AOS & OSA & AOS \\
\hline $\begin{array}{l}\text { Unähnliche Gruppe: } \\
\text { Männer }\end{array}$ & 8 & 8 & 8 & 8 \\
\hline $\begin{array}{l}\text { Ahnliche Gruppe: } \\
\text { Frauen }\end{array}$ & 8 & 8 & 8 & 8 \\
\hline Keine Gruppe & 8 & 8 & 8 & 8 \\
\hline
\end{tabular}

terschiedliche Studienfächer. Die Vpn waren im Mittel im vierten Semester. Sie erhielten DM 8. - für die Teilnahme.

Versuchsablauf. Bei ihrem Eintreffen wurde jede $\mathrm{Vp}$ schriftlich darüber informiert, daß es um das Thema «Gleichberechtigung der Frau» gehe und daß ausschließlich Frauen untersucht würden, da bereits eine Untersuchung an Männern erfolgt sei. Jede Vp erhielt neun Karten mit je einer kurzen Feststellung in Stichwortform, die einen Vorschlag zur Lösung der Frage der Gleichstellung von Frauen betraf. (Diese Feststellungen waren aufgrund von Vorversuchen so ausgewählt worden, daß sie nicht allzu stark unterschiedlich präferiert wurden). Die Vorschläge lauteten:

1. Gleiche Ausbildung für Männer und Frauen

2. Keine geschlechtsspezifische Erziehung

3. Mehr Institutionen, die Müttern die Sorge um Kinder abnehmen

4. Mehr leitende Positionen für Frauen

5. Gerechte Aufteilung der Pflichten im Haushalt

6. Mehr Teilzeitarbeitsplätze für Männer und Frauen

7. Förderung der Berufstätigkeit von Frauen mit Kindern

8. Bezahlung der Hausfrauenarbeit an die Hausfrau

9. Mehr Hausarbeit für Männer

Bedingung «Wahlfreiheit» («Commitment»): Die Vp sollte die Karten in einer Rangreihe danach legen, wie sie meint, wie dringlich bzw. wichtig die Vorschläge sind, um eine Gleichstellung der Frau zu erreichen.

Bedingung «Keine Wahlfreiheit» ( No Commitment»): Die Vp bekam eine Rangreihe der Karten vorgelegt (und zwar jeweils diejenige, die die zuvor untersuchte Person unter der Bedingung «Wahlfreiheit» gelegt hatte, mit der Bemerkung «Diese Rangreihe von Vorschlägen, um eine Gleichstellung der Frau zu erreichen, hat eine Kommilitonin gelegt. Gib bitte eine Schulnote als Bewertung an, wie Du diese Rangreihe bewertest!m

Bedingung «Selbstaufmerksamkeit» (OSA): Die Vp wurde in einen größeren Versuchsraum geführt, in dem eine Video-Mitschauanlage steht; die Kamera ist auf die Vp gerichtet, und die Vp kann ihr Bild auf dem Videoschirm verfolgen. Die Vp setzt sich schräg gegenüber an den Tisch und wird gebeten, eine Fallgeschichte durchzulesen. Nach dem Durchlesen soll sie ihren Lösungsvorschlag auf ein Blatt möglichst kurz aufschreiben und dann laut so vorlesen, $\mathrm{da} B$ er auf Videoband aufgenommen wird. 
Bedingung «Geringe Selbstaufmerksamkeity $(A O S)$ : Die Vp wird in den gleichen Raum geführt, doch ist hier die Kamera zur Wand gedreht, und die Videoanlage ist sichtbar abgeschaltet; die Geräte stehen offensichtlich nur zufällig im Raum. Die Fallgeschichte soll lediglich kurz schriftlich beantwortet werden.

\section{Die zu beurteilende Fallgeschichte lautet:}

«Elisabeth und Frank sind nun seit knapp zwei Jahren verheiratet. E., die Germanistik und Französisch studiert hat, hat vor wenigen Monaten mit ihrer Referendarzeit angefangen, die sie zwar als sehr anstrengend und nervenaufreibend empfindet, die ihr aber auch aufgrund der durch die Schüler erfahrenen Bestätigung viel Spaß macht. Sie hat vor, die Referendarzeit zu Ende zu machen und dann bis zu ihrer Beamtung auf jeden Fall als Lehrerin tätig zu sein. Ihr Mann Frank arbeitet seit zwei Jahren als Jurist in einer Rechtsanwaltsprax is und hat dort gute Aussichten, bald als gleichberechtigter Rechtsanwalt in die Praxis einzusteigen.

In dieser Situation erfahrt E. von ihrem Frauenarzt, daß sie schwanger sei. Obwohl beide in einigen Jahren sich Kinder gewünscht hätten, trifft sie diese Nachricht doch sehr unerwartet, weil sie die Zukunftspläne beider durcheinander wirft, und es nun gilt, gemeinsam mit dieser $\mathrm{Si}$ tuation fertig zu werden. (Abtreibung kommt für beide nicht in Frage, so daß eine andere Lösung gefunden werden muß.)

Für Frank scheint die ganze Situation gar nicht so problematisch zu sein, denn für ihn bietet sich eigentlich nur eine diskutable Lösung an. Aufgrund seiner guten Erfolgsaussichten, die ihm in der Praxis geboten werden, sieht er für seine Frau gar nicht die Notwendigkeit, ihre Ausbildung fortzusetzen, da er so gut verdient, daß sie sich in aller Ruhe nur um Kind und Haushalt kümmern kann. Eine Doppelbelastung würde er seiner Frau ja sowieso nicht zumuten wollen.

Elisabeth dagegen ist mit dieser Lösung ganz und gar nicht einverstanden. Sie möchte ihre Ausbildung auf jeden Fall fortsetzen, da sie sich nicht vorstellen kann, in der Mutter- und Hausfrauenrolle ganz aufzugehen. Das Kind ist für sie kein Grund, ihre ganzen Zukunftspläne aufzugeben und damit auch ihre Eigenständigkeit, sowohl finanziell als auch als Erfahrungs- und Betätigungsfeld außerhalb der engen Welt von Heim und Herd. Sie sieht die einzige Lösung darin, daß Frank nur noch halbtags arbeitet und auf das Kind aufpaßt, derweil sie ihre Ausbildung vormittags fortsetzt.

F. ärgert sich sehr, daß seine Frau kein Verständnis aufbringt, daß die Umsetzung ihrer modernistischen Ideen für ihn gar nicht realisierbar ist. Er würde sich sowieso lächerlich machen, wenn er seinen Kollegen sagen würde, er könne in Zukunft nur noch halbtags arbeiten, da er Hausmann spielen müsse, da er statt seiner Frau auf das Kind aufpassen müsse. Auch wären seine Aufstiegschancen damit gewiß so gut wie gestorben. Steuerlich stelle es sowieso nur einen Nachteil dar, wenn E. weiterarbeiten wolle, da der Staat bei Doppelverdienern übermäßig viele Steuern einziehe. Auch habe er in allen Zeitschriften gelesen, daß es für das Kind sehr wichtig sei, eine feste Bezugsperson zu haben, und das sei bei diesem ständigen Wechsel zwischen vormit tags und nachmit tags bestimmt nicht gewährleistet.

E., die mit ihrem Beruf auch eine gewisse Selbstverwirklichung verbunden sieht, kann diese Vorschläge nicht akzeptieren. Warum soll gerade sie diejenige sein, für dic sich durch das Kind alles verändert?»

Anschließend an die Beurteilung der Fallgeschichte legte der Vlder Vp wiederum die neun Karten zur Beurteilung vor. Die Karten wurden jeder Vp in einer Rangreihe vorgelegt, die (bis auf die Extreme) der genau umgekehrten Reihenfolge, wie sie die $\mathrm{Vp}$ im ersten Durchgang gelegt hatte (unter der «Commitment»-Bedingung) oder vorgelegt bekommen hatte (unter der «No Commitment»-Bedingung), entsprach. (Z.B. wenn die erstgelegte Rangfolge «2, 4, 3, 1, 6, 5, 9, 7, 8» lag, dann wurde nun die Folge $\ll 7,8,9,5,6,1,3,2,4 »$ vorgelegt.) Dies geschah unter den folgenden beiden Versuchsbedingungen:

Bedingung «Männlicher Gruppenstandard» ( (Dissimilar Group»): Der Vp wurde gesagt, die nun vorgelegte Rangreihe sei diejenige von 96 Männern, die vor kurzem untersucht worden seien, d.h., es handle sich um die mittlere bzw. typische Rangreihe der Lösungsvorschläge dieser Männer.

Bedingung "Weiblicher Gruppenstandard» ("Similar Group»): Der Vp wurde gesagt, bei der vorgelegten Rangreihe handle es sich um die mittlere bzw. typische Rangreihe der Lösungsvorschläge von 96 Frauen, die zuvor untersucht worden seien.

(Unter der Bedingung "Kein Gruppenstandard» bzw. "No Group» wurde keine solche weitgehend invertierte - Rangreihe vorgegeben.)

Schließlich erhielten alle Vp die folgende Instruktion:

«Nachdem Du Dich jetzt mit der Geschichte auseinandergesetzt hast und (Fingerzeig auf die vorgelegte Rangreihe; dies jedoch nicht unter der (No Groups-Bedingung) diese Information erhalten hast (letzterer Halbsatz entfiel unter der (No Group)-Bedingung), bitten wir Dich nun, die Karten noch einmal in eine Rangreihe nach Deiner persönlichen Priorität zu ordnen.»

Abhängige Variablen. Als erste abhängige Variable (für den $2 \times 2 \times 3$-Versuchsplan, also unter Einschluß der Personen, die nicht dem Einfluß eines «Gruppenstandards» ausgesetzt wurden) wurde die Rangreihenkorrelation zwi- 
schen der ersten (selbst gelegten oder vorgelegten) und der letzten (selbstgelegten) Rangreihe der Lösungsvorschläge pro Person berechnet und in den entsprechenden Standardwert transformiert (standardisierte Korrelation $z_{1}$ ). Als zweite abhängige Variable (für den $2 \times 2 \times 2$-Versuchsplan) wurde die entsprechende standardisierte Rangreihenkorrelation zwischen der zweiten (angeblich von der Gruppe männlicher oder weiblicher Personen gelegten) und der letzten (selbstgelegten) Rangreihe der Lösungsvorschläge pro Person berechnet (standardisierte Korrelation $z_{2}$ ). Während $z_{1}$ in gewissem Maße (zumindest bei Wahlfreiheit) als $\mathrm{Maß}$ der «Selbstkonsistenz» aufgefaßt werden kann, läßt sich $z_{2}$ als Maß der «Gruppenkonformität» auffassen.

Nachbefragung. Jede $\mathrm{Vp}$ füllte anschließend schriftlich und ohne Aufsicht durch den Versuchsleiter einen Fragebogen mit Fragen zu dem voraufgegangenen Experiment aus (vgl. Manipulationsüberprüfung).

Aufklärung über den Versuch. Zur Aufklärung über das Experiment wurde ein Besprechungstermin nach dem Ende des letzten Einzelversuchs angeboten.

\section{Ergebnisse}

Manipulationsüberprüfung. Auf die bei der Nachbefragung gestellte Frage, ob der Vp bewußt gewesen sei, warum sie als $\mathrm{Vp}$ fungiert habe, gaben $87 \%$ sinngemäß an, daß es um weibliche $V$ pn gegangen sei. (Der überwiegende Teil der Vpn schien sich somit bewußt zu sein, als Frau angesprochen zu werden.) Auf die Frage, ob die Vp den Eindruck hatte, bei der Lösung der Fallgeschichte beobachtet zu werden, antworteten unter der OSA-Bedingung 37, unter der AOS-Bedingung sechs von jeweils 48 Vpn mit «Ja» bzw. dem Hinweis auf die Videoanlage $\left(\mathrm{chi}^{2}=40.49 ; \mathrm{df}=1 ; \mathrm{p}<0.001\right)$. (Unter der OSA-Bedingung fühlte sich demnach im Gegensatz zur AOS-Bedingung der überwiegende Teil der Vpn während des Versuchs «beobachtets.) Auf die Frage, von welcher Personengruppe die Vp eine Rangreihe von Vorschlägen zur Lösung der Frauenfrage vorgelegt bekommen habe, gaben unter den Bedingungen «Ähnliche/Unähnliche/Keine Gruppe»12/8/
Tab. 2: Zellenmittelwerte der abhängigen Variablen $\mathrm{z}_{1}$.

\begin{tabular}{lllll}
\hline & \multicolumn{2}{l}{ Commitment } & \multicolumn{2}{l}{$\begin{array}{l}\text { Kein } \\
\text { Commitment }\end{array}$} \\
\cline { 2 - 5 } & OSA & AOS & OSA & AOS \\
\hline Unähnliche Gruppe & 1.9 & 2.2 & 0.5 & 0.7 \\
\hline Ähnliche Gruppe & 1.8 & 1.7 & 0.9 & 0.4 \\
\hline Keine Gruppe & 1.9 & 1.4 & 0.8 & 1.0 \\
\hline
\end{tabular}

32 Personen eine falsche, und 20/24/0 Personen eine richtige Antwort $\left(\mathrm{chi}^{2}=41.62 ; \mathrm{df}=2\right.$; $\mathrm{p}<0.001$ ). (Der überwiegende Teil der Vpn konnte sich also richtig erinnern, welcher Gruppe die vorgelegte - weitgehend umgekehrte - Rangreihe von Lösungsvorschlägen zugeschrieben worden war.)

Ergebnisse für die abhängige Variable $z_{1}$ (Maß der «Selbstkonsistenz»). Die $2 \times 2 \times 3$ Varianzanalyse mit den Faktoren «Commitment» (abhängig), «Selbstaufmerksamkeit» und «Gruppeneinfluß» ergab lediglich einen auf dem 0.001 -Niveau signifikanten «Commitment»-Effekt, also einen Unterschied zwischen Personen mit und ohne Möglichkeit, selbständig eine Rangreihe von Lösungsvorschlägen zu geben. (Wir haiten diesen Effekt für trivial.) Es ergab sich jedoch keine interpretierbare Wechselwirkung irgendeiner Art, also auch nicht mit dem Faktor "Gruppe». Die Zellenmittelwerte des Planes mit der abhängigen Variablen $z_{1}$ sind in Tabelle 2 aufgeführt.

Ergebnisse für die abhängige Variable $z_{2}$ (Maß der «Gruppenkonformität»). Die $2 \times 2 \times 2$-Varianzanalyse mit den Faktoren «Commitment» (abhängig), «Selbstaufmerksamkeit» und «Gruppe» (hier nur zweistufig, da die «No-Group»-Bedingung logischerweise entfällt, wenn es um den Zusammenhang zwischen persönlichem und «Gruppen»-Urteil geht), ergab, wie Tabelle 3 im einzelnen zeigt, neben dem hochsignifikanten «Commitment»Effekt eine signifikante Wechselwirkung zwischen «Selbstaufmerksamkeit» und «Gruppeneinfluß». Tabelle 4 zeigt die Zellenmittelwerte von $\mathrm{z}_{2}$. Alle Korrelationen sind mehr oder weniger negativ; sie repräsentieren den Zusammenhang zwischen der (zumindest im Falle von Wahlfreiheit) zur eigenen Meinung konträren, einer Gruppe zugeschriebenen Präferenzenliste einerseits, und der selbsthergestellten Präferenzenliste andererseits. 
Tab.3: Varianzanalyse von $\mathrm{z}_{2}$ (standardisierte Korrelation zwischen «Gruppen-» und individueller Rangreihe der Präferenzen).

\begin{tabular}{|c|c|c|c|}
\hline Quelle & QUS df & MQUS 1 & $\mathbf{F}$ \\
\hline Selbstaufmerksamkeit & 0.03 & 0.03 & 0.11 .75 \\
\hline Gruppe & 0.15 & 0.15 & 0.53 .47 \\
\hline Selbstaufm $\times$ Gruppe & $1.42 \quad 1$ & 1.42 & $4.89 .04^{*}$ \\
\hline Error & 8.1528 & 0.29 & \\
\hline Commitment & 16.65 & 16.65 & $46.63 .00^{* * x}$ \\
\hline Commitm. $\times$ Selbstaufm & 0.48 & 0.48 & 1.35 .26 \\
\hline Commitm.$\times$ Gruppe & 0.28 & 0.28 & 0.79 .38 \\
\hline $\begin{array}{l}\text { Comm. } \times \text { Selbstaufm } . \\
\times \text { Gruppe }\end{array}$ & $0.14 \quad 1$ & 0.14 & 0.39 .54 \\
\hline Error & 10.0028 & 0.36 & \\
\hline
\end{tabular}

${ }^{*} \mathrm{p}<0.05,{ }^{* * *} \mathrm{p}<0.001$

Tab. 4: Zellenmittelwerte der abhängigen Variablen $\mathrm{z}_{2}$.

\begin{tabular}{lllll}
\hline Zellenmittelwerte & \multicolumn{2}{c}{ Commitment } & \multicolumn{2}{l}{$\begin{array}{l}\text { Kein } \\
\text { Commitment }\end{array}$} \\
\cline { 2 - 5 } & OSA & AOS & OSA & AOS \\
\hline Unähnliche Gruppe & -1.5 & $-2.0^{*}$ & -0.6 & -0.6 \\
\hline Ähnliche Gruppe & -1.6 & $-1.4^{*}$ & -0.8 & -0.4 \\
\hline \multicolumn{1}{c}{ Mit * versehene Mittelwerte bilden den einzigen signifi- } \\
kanten Kontrast, der nicht auf den Commitment-Faktor \\
zurückgeht.
\end{tabular}

Von den einzelnen Kontrasten weist der DUNCAN-Test als einzige Mittelwertsdifferenz, die nicht auf den Commitment-Faktor zurückzuführen ist, diejenige zwischen der «weiblichen» und der männlichen Gruppe bei Wahlfreiheit («commitment») und bei fehlender Selbstaufmerksamkeit (AOS) als statistisch gesichert aus. Der stärkste Gruppen-Einfluß zeigt sich somit unter der Bedingung, daß das Individuum nicht «objektiv selbstaufmerksam» ist.

Die gefundene Wechselwirkung zwischen den Faktoren «Objektive Selbstaufmerksamkeit» (OSA vs. AOS) und «Gruppeneinfluß» («Ähnliche Gruppe» = SIM = Similar Group bzw. Ingroup vs. «Unähnliche Gruppe» = DIS $=$ Dissimilar Group bzw. Outgroup) ist in Abbildung 1 graphisch dargestellt. (Dabei ist $\mathrm{zu}$ beachten, daß das Maß des Gruppen-Einflusses auf die Meinungen des Individuums umso geringer ist, je numerisch höher die negativen Korrelationen zwischen Gruppen-Rangreihe und individueller Rangreihe ausfallen, d.h., je höher die $z_{2}$-Werte, also je niedriger die Abszissenwerte sind.)

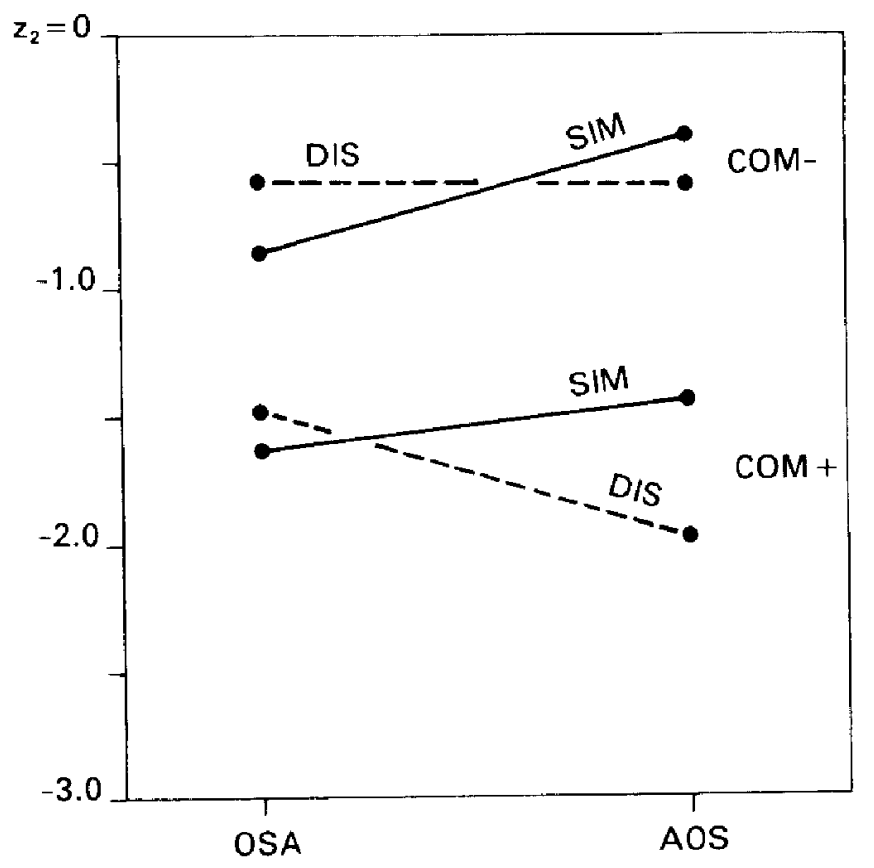

Abb. 1: Graphische Darstellung der Wechselwirkung von aSelbstaufmerksamkeit» (OSA/AOS) und "Gruppeneinfluß» (SIMilar/DISsimilar Group) auf die Gruppenkonformität $\left(\mathrm{z}_{2}\right)$ bei unterschiedlichen Graden von Wahlfreiheit (COMmitment $+/-$ ).

\section{Diskussion}

Die Annahme, daß Personen, die eine Einstellung frei geäußert haben, im Zustande «objektiver Selbstaufmerksamkeit» unabhängig von den Einstellungen der Gruppe Selbstkonsistenz zeigen, konnte aufgrund der vorliegenden Untersuchung nicht bestätigt werden. Das Experiment lieferte vielmehr empirische Unterstützung für die Hypothese, daß es zu der von WICKLUND bzw. McCoRMICK postulierten «Unabhängigkeit von der Gruppen-Einstellung» eher dann kommt, wenn es sich um die Beziehung zwischen einem Individuum und einer diesem Individuum unähnlichen Gruppe (bzw. einer Outgroup) handelt, als wenn es um eine Individuum-Ingroup-Beziehung geht. Abweichend von den genannten Autoren zeigte sich dies jedoch nicht unter OAS-, sondern unter AOS-Bedingungen, also vornehmlich dann, wenn die Person nicht besonders selbstaufmerksam ist. Die Interpretation MCCoRMICKs, daß ein «freies und selbstaufmerksames» Individuum zur Selbstkonsistenz fähig und damit 
ein positives Ziel pädagogischer Bemühungen sein könne (1979, p.61), erscheint im Lichte der vorliegenden Ergebnisse zumindest verfrüht. Es ist allerdings zu fragen, ob das vorliegende Experiment zur Überprüfung der McCorMICK- bzw. WICKLUNDschen Annahmen geeignet und als teilweise Replikation der MCCORMICKschen Untersuchung anzusehen ist; gleichzeitig ist zu diskutieren, ob in einem Versuch dieser Art wirklich stets «Selbstkonsistenz» erfaßt wurde.

Um mit dem Zuletztgenannten zu beginnen: Die von McCormick vorgenommene Operationalisierung von «Free Choice» bzw. «Commitment», die wir der Replikation halber in dem vorliegenden Experiment wiederholt haben, erscheint angesichts dessen, was untersucht werden soll (und auch angesichts oben schon erwähnter, weitreichender Schlüsse, die aus den experimentellen Ergebnissen gezogen werden), wenig sinnvoll. «Selbstkonsistenz» als Konsistenz zwischen dem Verhalten und der Einstellung einer Person (oder einer Einstellung zum Zeitpunkt 1 und zu einem späteren Zeitpunkt) läßt sich unseres Erachtens im vorliegenden Falle lediglich unter der «Free Choice» bzw. «Commitment»-Bedingung erfassen, da die Versuchspersonen ohne freie Wahlmöglichkeit bereits eine nicht selbst gelegte Rangreihe präsentiert bekamen, und gegenüber einer nicht selbst produzierten Präferenzenliste kann man ja schwerlich so etwas wie Selbstkonsistenz beweisen. Die Maße der «Selbstkonsistenz» $\left(\mathrm{z}_{1}\right)$ bzw. der «Gruppenkonformität» $\left(z_{2}\right)$ scheinen uns daher lediglich unter der Bedingung freier Wahlmöglichkeit ( «Commitment» bzw. «Free Choice») Validität zu besitzen. Die hochsignifikanten «Commitment»-Effekte für die abhängigen Variablen $z_{1}$ und $z_{2}$ erscheinen somit als nicht interpretierbar.

Ein wesentlicher Unterschied zwischen dem MCCORMICKschen und dem hier berichteten Experiment betrifft das Einstellungsobjekt. MCCORMICK verwendete, wie viele andere Experimentatoren, denen es darum geht, Einstellungsbildung und Einstellungsänderung zu untersuchen, Urteilsobjekte, die - verglichen mit den von uns herangezogenen - kaum die Bezeichnung (Einstellungsobjekt) verdienen; solche Gegenstände mögen zwar den Vorteil ha- ben, Prozesse der Meinungsgenese besser studieren $z u$ helfen, doch kann man sich schwer vorstellen, daß sie zur externen Validität eines Experimentes zur Änderung sozialer Einstellungen unter Gruppeneinfluß Wichtiges beitragen. Persönlich bedeutsamere Einstellungen wie solche zur «Gleichberechtigung» zu untersuchen, hieße nach unserer Auffassung, Tendenzen zur Selbstkonsistenz gegenüber solchen zur Gruppenkonformität zu begünstigen. WICKLUND (in einer persönlichen Mitteilung) ist hier anderer Auffassung: Gerade bei solchen sozial relativ irrelevanten Einstellungsobjekten wie den McCORMICKschen gebe es keinen zwingenden sozialen Grund für die Vp, warum ihre eigenen Präferenzen die gleichen wie diejenigen anderer Vpn sein sollten; abzuweichen bedeute nicht, sich «falsch» zu verhalten. WiCKLUND kommt also zu dem Schluß, daß solche bedeutsamen Einstellungsobjekte wie "Gleichberechtigung» eher Tendenzen zur Gruppenkonformität begünstigen würden. Wie immer die Antwort auf diese Frage ausfallen mag: Es erscheint uns notwendig, Experimente zur Änderung sozialer Einstellungen unter sozialem Einflu $B$ - falls dies, wie im vorliegenden Falle, technisch möglich ist - bevorzugt an $\mathrm{Ge}$ genständen vorzunehmen, die für den Einstellungsträger von tatsächlicher sozialer Bedeutung sind.

Eine weitere, vermutlich weniger gravierende Abweichung der vorliegenden Operationalisierung von der Vorlage McCoRMicks betrifft die Herstellung von «objektiver Selbstaufmerksamkeit» und die Angemessenheit der Manipulationsüberprüfung. Zwar waren die Ergebnisse unserer postexperimentellen Befragung in allen Fällen positiv, doch läßt sich gegen solche Befragungen eine Vielzahl von Einwänden erheben, z. B. derjenige, daß die «richtigen» Antworten erst nachträglich induziert werden. Noch gewichtigere Einwände, so scheint es uns, lassen sich jedoch gegen die Anwendung eines Fragebogens wie der Scale of Private Self-Consciousness von FENigstein, SCHEIER \& Buss (1975) erheben, da damit gerade auf eher habituelle als aktuelle, d.h. durch experimentelle Manipulation induzierte Selbstbeurteilung abgehoben wird. Die Unvollkommenheit von Manipulation Checks der objektiven Selbstaufmerksamkeit scheint somit ein allgemeines, 
nicht ein spezielles Problem der vorliegenden Untersuchung zu sein (vgl. WICKLUND, 1975, pp. $267 \mathrm{ff}$.).

Das wichtigste Resultat der vorliegenden Untersuchung besteht darin, daß sich bei freier Wahlmöglichkeit - und hier wiederum im $\mathrm{Zu}$ stande fehlender objektiver Selbstaufmerksamkeit - ein differentieller Einfluß der Art der sozialen Gruppe auf die Einstellung des Individuums nachweisen läßt, und zwar je nach der Beziehung zwischen Individuum und Gruppe in Relation zu dem in Frage stehenden Einstellungsobjekt. Bei nicht besonders auf die eigene Person gelenkter Aufmerksamkeit scheinen die gleichsam automatisierten Effekte «Übereinstimmung mit der Ingroup bzw. der ähnlichen Gruppe» und «Ablehnung der Meinungen der Outgroup bzw. der unähnlichen Gruppe» aufzutreten - diese Differenzierung findet bei objektiver Selbstaufmerksamkeit nicht statt. Die Abhebung bzw. Distanzierung von der Gruppenmeinung, und damit der geringste Einflu $B$ der sozialen Gruppe, findet nicht unter der Bedingungskombination «Selbstaufmerksamkeit und Wahlfreiheit» (wie bei MCCORMICK) statt, sondern unter der Bedingungskombination «Fehlende Selbstaufmerksamkeit, Wahlfreiheit und Outgroup-Einfluß) - handelt es sich dagegen um eine Gruppe, der sich die Person zugehörig fühlen kann, was den Einstellungsgegenstand betrifft, so spielt der Grad ihrer Selbstaufmerksamkeit für das Ausmaß des Gruppeneinflusses keine Rolle.

Dieses Ergebnis widerspricht nicht nur den Befunden von MCCormick (1979), sondern auch denjenigen von DUVAL \& WICKLUND (1971), da die Analyse der Wechselwirkung von «Selbstaufmerksamkeit» und "Gruppeneinfluß» auf das Ausmaß von Gruppenkonformität einen entgegengesetzen OSA-Effekt ergibt. Der von uns gefundene $A O S$-Effekt läßt sich jedoch ohne weiteres sozialpsychologisch sinnvoll interpretieren, wenn man - wie etwa in der Theorie der Intergruppenbeziehungen von TAJFEL geschehen - vom differentiellen Einflu $\beta$ unterschiedlicher Arten von Person-GruppenBeziehungen ausgeht.

Gerade dann, wenn ein Individuum nicht besonders selbstaufmerksam ist, wird sich die mehroder weniger unreflektierte Tendenz, sich der Ingroupmeinung anzuschließen und die
Outgroupmeinung zurückzuweisen, bei der Person durchsetzen - eine andere Erklärung für das Ergebnis, daß sich der geringste Gruppeneinfluß unter der Bedingungskombination AOS/Outgroup zeigt, können wir nicht anbicten. In Übereinstimmung mit TAJFELS Theorie der Intergroup Differentiation sowie TAJFEI \& TURNERs Theorie der Social Identity lassen sich die Effekte der verschiedenen Kombinationen der Bedingungen OSA, AOS, In- und Outgroup auf die Gruppenkonformität wie folgt interpretieren: Die gleichsam automatisch und unreflektiert vorgenommene Differenzierung zwischen der einstellungsmäßig ähnlichen und unähnlichen Gruppe (also so etwas wie ein alltäglicher Ingroup/Outgroup-Bias in bezug auf soziale Einstellungen und Meinungen) verschwindet dann, wenn das Individuum in den Zustand der objektiven Selbstaufmerksamkeit versetzt wird - möglicherweise aus dem Grunde, daß dann übergeordnete Standards wie derjenige, sich nicht vom «irrationalen» GruppenBias leiten zu lassen, salient werden. In Termini von TAJfels Kontinuum sozialer Interaktionen, das ein interpersonales und ein InterGruppen-Ende aufweist, ausgedrückt, heißt dies: Beim Übergang vom Zustand der objektiven Selbstaufmerksamkeit (OSA) in den $\mathrm{Zu}$ stand der Aufmerksamkeit von der eigenen Person weg (AOS) bewegt sich das Individuum kognitiv von der interpersonalen hin zur InterGruppen-Ebene der sozialen Interaktion, auf der stereotype Wahrnehmungen der IngroupÄhnlichkeit und der Outgroup-Unähnlichkeit gegenwärtig sind.

Diese Interpretation stellt $u$.E. nicht die Bedeutung von Prozessen der Selbstaufmerksamkeit für solche der sozialen Beeinflussung in Frage, wenn sie auch einstweilen inkonsistent mit bisherigen Feststellungen zur Theorie der objektiven Selbstaufmerksamkeit sind. Sie weist vielmehr aufdie Notwendigkeit hin, Konzepte des "Gruppeneinflusses» person- und einstellungsspezifisch zu differenzieren und über die Formulierung von Annahmen zur «objektiven Selbstaufmerksamkeit» hinaus die Theoriebildung auf weitere Aspekte der Relation zwischen dem Individuum und seinem sozialen Kontext auszudehnen. 


\section{Literatur}

Carver, C.S. 1975. Physical aggression as a function of objective self awareness and attitudes towards punishment. Journal of Experimental Social Psychology, 11, 510519.

Duval, S. 1972. Conformity as a function of perceived level of personal uniqueness and being reminded of the object status of self. Unpublished doctoral dissertation, University of Texas (zit. n. Wicklund, 1980).

Duval, S. 1976. Conformity on a visual task as a function of personal novelty on attitudinal dimensions and being reminded of the object status of self. Journal of Experimental Social Psychology, 12,87-98.

Duval, S. \& WickLUND, R.A. 1972. A theory of objective self-awareness. New York: Academic Press.

Fenigstein, A., Scheier, M. F. \& Buss, A.H. 1975. Public and private self-consciousness: Assessment and theory. Journal of Consulting and Clinical Psychology, 43, $522-527$.

GibBons, F.X. 1978. Sexual standards and reactions to pornography: Enhancing behavioral consistency through self-focused attention. Journal of Personality and Social Psychology, 36, 976-987.
MCCoRmick, T.F. 1979. An investigation of standards of correctness by inducing conformity and consistency pressures within the framework of objective self awareness. Unpublished doctoral dissertation, University of Texas.

TAJFEL, H. \& TURNER, J. 1979. An integrative theory of intergroup conflict. In: Austin, W.G. \& Worchel, S. (Eds.): The social psychology of intergroup relations. Monterey, Cal.: Brooks/Cole.

Wicklund, R.A. 1980. Group contact and self-focused attention. In: Paulus, P. B. (Ed.): Psychology of group influence. Hillsdale, N.J.: Erlbaum.

Wicklund, R.A. \& Duval, S. 1971. Opinion change and performance facilitation as a result of objective self awareness. Journal of Experimental Social Psychology, $7,319-342$.

Die Verfasser danken Dipl. Psych. Heinz-Gerd Bolten für seine Tätigkeit als Versuchsleiter und für Auswertungsarbeiten, ferner Birgitta Sticher für ihre Mitarbeit bei der Erstellung des Versuchsmaterials. 